\title{
Investigation of Defect Characteristics and Carrier Transport Mechanisms in GaN Layers With Different Carbon Doping Concentration
}

\author{
Hongyue Wang ${ }^{\circledR}$, Member, IEEE, Po-Chun Hsu, Ming Zhao ${ }^{\circledR}$, Member, IEEE, \\ Eddy Simoen ${ }^{\circledR}$, Senior Member, IEEE, Arturo Sibaja-Hernandez, and Jinyan Wang ${ }^{\circledR}$, Member, IEEE
}

\begin{abstract}
In this article, a metal/carbon-doped GaN (GaN:C)/Si-doped GaN (GaN:Si) structure was used to investigate the defect characteristics and carrier transport mechanisms in GaN:C layers with different carbon doping concentration. Capacitance-voltage, current-voltage, and deep-level transient spectroscopy measurements were performed at different temperatures. At forward bias, a pinning effect was found at the interface of the GaN:C/GaN:Si layer, due to the defects capturing electrons. The forward currents of the samples with high carbon doping concentration $\left(N_{C}>1 \times 10^{19} \mathrm{~cm}^{-3}\right)$ increase gradually with increasing forward bias voltage. Ohm's law, space-charge-limited current, and variable-range-hopping mechanisms may dominate the forward current. For the samples with low carbon doping concentration $\left(N_{C}<1 \times 10^{19} \mathrm{~cm}^{-3}\right)$, a device turning on behavior was observed, which is attributed to the carriers overcoming a potential barrier. In addition, the DLTS spectra reveal that only electron trapping happens at forward bias for the samples with high carbon doping concentration, while, in addition, hole trapping was observed for the samples with low carbon doping concentration. The process of the carrier capture by defects was demonstrated.
\end{abstract}

Manuscript received August 8, 2020; accepted September 10, 2020. The review of this article was arranged by Editor S. Chowdhury. (Corresponding author: Hongyue Wang.)

Hongyue Wang is with the Science and Technology on Reliability Physics and Application of Electronic Component Laboratory, China Electronic Product Reliability and Environmental Testing Research Institute, Guangzhou 510610, China, also with the IMEC, 3001 Leuven, Belgium, and also with the Department of Chemistry, Katholieke Universiteit Leuven, 3001 Leuven, Belgium (e-mail: wanghonghyue@pku.edu.cn).

Po-Chun Hsu is with IMEC, 3001 Leuven, Belgium, and also with the Department of Materials Engineering, Katholieke Universiteit Leuven, 3001 Leuven, Belgium (e-mail: brent.hsu@imec.be).

Ming Zhao and Arturo Sibaja-Hernandez are with IMEC, 3001 Leuven, Belgium (e-mail: ming.zhao@imec.be; arturo.sibayahernandez@imec.be).

Eddy Simoen is with IMEC, 3001 Leuven, Belgium, and also with the Department of Solid State Science, Ghent University, 9000 Ghent, Belgium (e-mail: eddy.simoen@imec.be).

Jinyan Wang is with the School of Electronics Engineering and Computer Science, Peking University, Beijing 10071, China (e-mail: wangjinyan@pku.edu.cn).

Color versions of one or more of the figures in this article are available online at http://ieeexplore.ieee.org.

Digital Object Identifier 10.1109/TED.2020.3025261
Index Terms-Carbon doping, defects, GaN buffer, transport mechanism.

\section{INTRODUCTION}

$\mathbf{R}$ ECENTLY, GaN-on-Si has become one of the most promising candidates for high-power and high-frequency applications [1]-[3]. Thanks to the epitaxy on 8-in largediameter $\mathrm{Si}$ substrates, the cost can be significantly reduced [4], [5]. However, the mismatch of lattice and thermal expansion between $\mathrm{Si}$ and $\mathrm{GaN}$ requires a strain management layer [transition layer (TL)] to control the wafer warp. Above the TL, a GaN buffer layer is grown, which is significant for high-power and RF devices due to its impact on the vertical leakage, the breakdown behavior, and the current collapse (CC). Carbon doping is usually employed to achieve a high resistivity GaN buffer layer, which, reportedly, can compensate the initial n-type background doping of $\mathrm{GaN}$, resulting in a semi-insulating layer [6], [7]. However, the carbon doping also induces defects to the $\mathrm{GaN}$ layer, which may capture electrons under OFF-state switching and degrade the ONstate resistance. Therefore, it is significant to adopt a proper carbon doping concentration to control the leakage current and avoid the CC. Some characterization techniques, such as photoluminescence [8]-[10], deep-level transient spectroscopy (DLTS) [11], and back-gate stressing [12], were employed to detect the defects located at different energy levels induced by the carbon doping. However, the impact of the carbon doping concentration on the defects and carrier transport mechanism still lacks detailed investigation. Different carrier transport mechanisms, including space-charge-limited current (SCLC) [13]-[15], hopping [16], [17], and Poole-Frenkel (PF) emission [18], [19], were proposed by various groups to interpret charge conduction in $\mathrm{GaN}$ :C. However, the traditional high-electron-mobility transistor (HEMT) structure makes it difficult to separate the channel and TLs' influence on the $\mathrm{GaN}$ :C buffer layer, hindering the full understanding of the physics model of the $\mathrm{GaN}: \mathrm{C}$ buffer layers.

Recently, Koller et al. [20] demonstrated a simple structure to gain more insight into this GaN:C layer, which can 


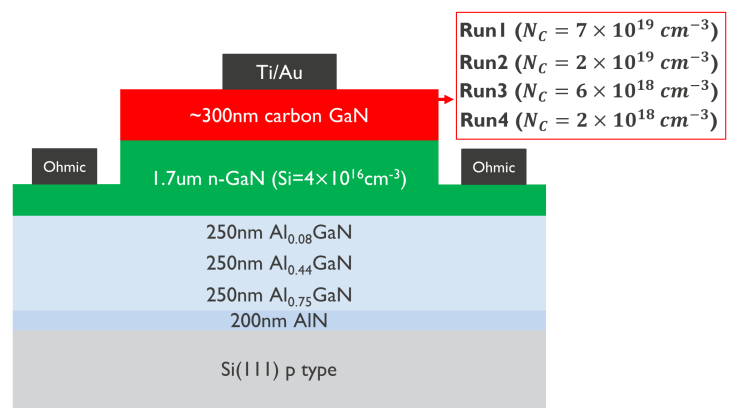

Fig. 1. Cross-sectional schematic of the devices fabricated on the four samples.

eliminate the influence of the channel layers. Here, we used a similar structure and fabricated Metal/GaN:C/GaN:Si devices to analyze the defect characteristics and carrier transport mechanisms of the GaN:C layer. Four samples with a $\mathrm{GaN}$ layer doped with different carbon concentrations were fabricated. $C-V, I-V$, and DLTS measurements were performed. Significantly different defect characteristics and carrier transport mechanisms between GaN:C layers with low and high carbon concentration doped were found. Furthermore, the band diagram of the samples under forward bias was derived, explaining the defect charging process and carrier transport behavior of the structure.

\section{Material Growth and Device Fabrication}

In this work, 4 wafers were epitaxially grown on 6-in p-type $\mathrm{Si}$ (111) substrates with a resistivity of $1-150 \Omega \mathrm{cm}$ using a Veeco turbodisc maxbright metal-organic chemical vapor deposition (MOCVD) system. The structure is shown in Fig. 1. At first, a 200-nm AlN nucleation layer was grown directly on the top of the Si substrate. Then, several AlGaN layers as TLs were grown on top of it, followed by a $1.7 \mu \mathrm{m}$ n-type GaN with $\sim 4 \times 10^{16} \mathrm{~cm}^{-3}$ silicon doping concentration. Then, a GaN layer with a nominal thickness of $300 \mathrm{~nm}$ and with different carbon doping concentration was grown by changing the growth temperature. The carbon doping concentrations $N_{C}$ are: $\sim 7 \times 10^{19} \mathrm{~cm}^{-3}$ (named Sample Run1), $\sim 2 \times 10^{19} \mathrm{~cm}^{-3}$ (Run2), $\sim 6 \times 10^{18} \mathrm{~cm}^{-3}$ (Run3), and $\sim 2 \times 10^{18} \mathrm{~cm}^{-3}$ (Run4), which were measured by secondary ion mass spectrometry (SIMS) from the samples. The thickness of the GaN:C layer shows a slight difference between these four samples due to the different growth conditions required for achieving the target carbon doping concentration. The actual GaN:C thicknesses of the four samples extracted from the in situ reflectivity measurement are 285, 278, 275, and $268 \mathrm{~nm}$, respectively. The Si doping concentration of the GaN:Si layer was also confirmed by SIMS measurement and an active electron density of $\sim 4 \times 10^{16} \mathrm{~cm}^{-3}$ was obtained from Hall measurement from a reference sample without the $\mathrm{GaN}: \mathrm{C}$ layer on top of the $\mathrm{GaN}$ :Si layer.

To avoid the impact of the TL/substrate and to have a direct insight of the GaN:C layer, a semivertical diode was fabricated with top-top contacts. Device fabrication commenced with isolation region etching. A 600-nm deep mesa was etched by $\mathrm{Cl}_{2}$-based inductively coupled plasma to expose the GaN:Si layer. Then, the ring-shaped ohmic contact metal

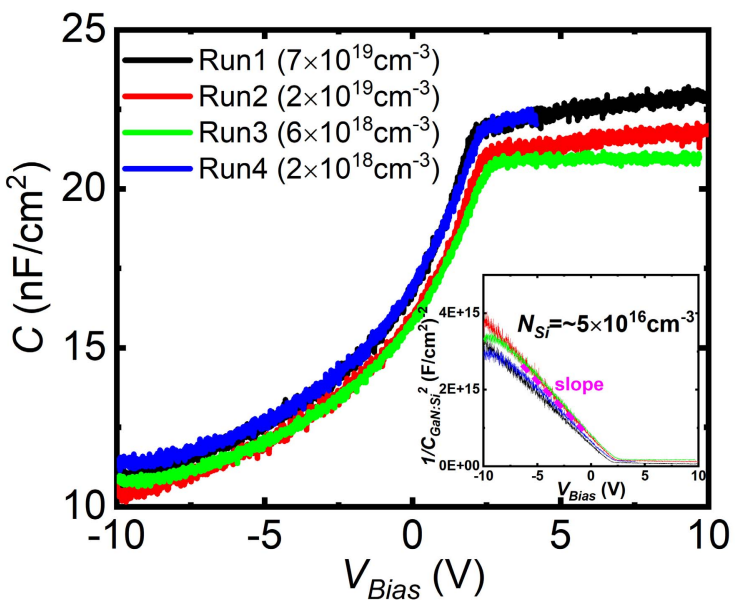

Fig. 2. $\quad C-V$ characteristics of samples Run1-Run4 with an ac signal frequency of $1 \mathrm{MHz}$. Inset: $1 / C_{\mathrm{GaN}: \mathrm{Si}}^{2}$ versus $V_{\text {Bias }}$ curves of samples Run1-Run4.

stack Ti/Al/Ti/Au (20/100/20/60 nm) was evaporated on it, followed by annealing at $550{ }^{\circ} \mathrm{C}$ for $90 \mathrm{~s}$ in $\mathrm{N}_{2}$ ambient. The ohmic contact resistance is around $2 \Omega \mathrm{mm}$, determined by the ring transfer length method. At last, the circular-shaped anode metal contacts of $200 \mu \mathrm{m}$ in diameter were fabricated by evaporation of a Ti/Au $(50 / 200 \mathrm{~nm})$ bilayer. Fig. 1 shows the cross-sectional schematic of the devices.

In order to investigate the carrier transport mechanism and defect characteristics of the carbon-doped GaN layer with different carbon doping concentration, capacitance-voltage $(C-V)$, current-voltage $(I-V)$, and DLTS measurements were performed.

\section{Results AND Discussion}

\section{A. Capacitance-Voltage Characteristics}

The room temperature $C-V$ measurements were conducted between the anode and ohmic contacts with an ac signal frequency of $1 \mathrm{MHz}$, which is high enough to ensure that the corresponding time is faster than the trap response time or time constant due to the parallel conductivity. The bias voltage was swept from -10 to $10 \mathrm{~V}$ (Run1, Run2, and Run3)/5 V (Run4), with a slow sweep ramp rate of $50 \mathrm{mV} / \mathrm{s}$, ensuring the trap occupancy to follow the dc bias voltage. The maximum forward bias voltages are different because the Run1-Run4 devices become conductive at higher bias voltage.

The $C-V$ curves for the four samples are shown in Fig. 2. All the samples show depletion like $C-V$ behavior for a $V_{\text {Bias }}$ below around $2.7 \mathrm{~V}$, and a capacitance plateau behavior when $V_{\text {Bias }}$ is above $2.7 \mathrm{~V}$ bias. Taking account of the plateau capacitance and low conductance under forward bias, the $\mathrm{GaN}: \mathrm{C}$ layer is seen as a semi-insulting layer. The measured small-signal capacitance $C$ of Run1-Run4 can be expressed as a series connection of $C_{\mathrm{GaN}: \mathrm{Si}}$ and $C_{\mathrm{GaN}: \mathrm{C}}$ [20]

$$
\frac{1}{C}=\frac{1}{C_{\mathrm{GaN}: \mathrm{Si}}}+\frac{1}{C_{\mathrm{GaN}: \mathrm{C}}} \text {. }
$$

By deriving the $1 / C_{\mathrm{GaN}: \mathrm{Si}}^{2}$ versus $V_{\mathrm{Bias}}$ curves, the $\mathrm{Si}$ doping concentration of the n-type GaN layer is $4.9-5.8 \times 10^{16} \mathrm{~cm}^{-3}$, which is consistent with the target value, indicating that the capacitance depletion result from the n-type $\mathrm{GaN}$ layer. 

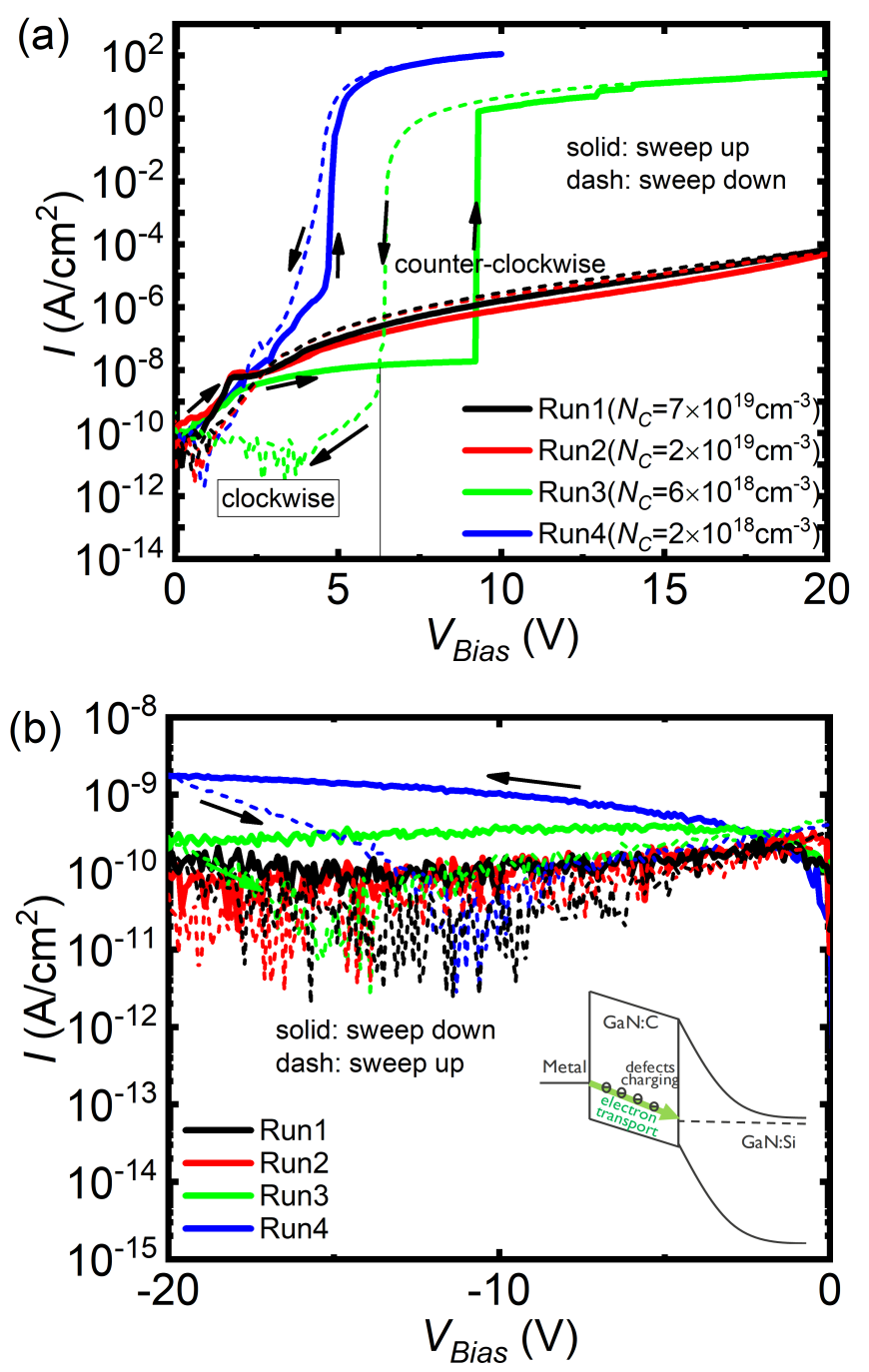

Fig. 3. Double sweep (a) forward and (b) reverse $I-V$ characteristics of the samples Run1-Run4 at room temperature.

\section{B. I-V Characteristics}

Double sweep $I-V$ measurements were performed on the four samples at room temperature. The reverse and forward $I-$ $V$ measurements were conducted separately to eliminate the impact of the forward and reverse bias on each other, giving rise to a "memory" effect by charge trapping and slow trap response. The forward bias was swept from 0 to $20 \mathrm{~V}$ and then back to $0 \mathrm{~V}$ with a voltage ramp rate of $100 \mathrm{mV} / \mathrm{s}$. The reverse bias was swept from 0 to $-20 \mathrm{~V}$ and then back to 0 $\mathrm{V}$ with the same voltage ramp rate.

Fig. 3(a) shows the forward $I-V$ curves of the samples from Run1 to Run4. The Run1 and Run2 show similar behavior, with a fast current increase before $1.5 \mathrm{~V}$ followed by a plateau, and then a gradual increase at higher forward bias. Run 3 shows a similar $I-V$ behavior before $1.5 \mathrm{~V}$ as Run1 and Run2. However, when the bias voltage continues to increase, the forward current remains almost constant, and when the bias voltage reaches around $10 \mathrm{~V}$, the current shows a steep increase, which is likely to be a device turning-on behavior instead of a hard breakdown by checking the reproducibility of the $I-V$ behavior. Run4 shows a rapid current rise under forward bias voltage, suggesting a poor blocking ability at forward bias. The double sweep $I-V$ hysteresis is complicated, as shown in Fig. 3(a). All the four samples show a clockwise hysteresis under low forward bias voltage (Run1 and Run2: 0-2.7 V, Run3: 0-6.2 V, and Run4: 0-2.2 V). While with the forward bias voltage increasing, the $I-V$ curves show counterclockwise hysteresis, indicating hole capture in the devices in this region. Note the hysteresis of Run3 and Run4 tends to decrease when increasing the ramping time (not shown here). This time-related turn-on behavior might relate to bistability between the ON-state filamentary conduction [21] and SCLC. The complicated forward $I-V$ behavior can be interpreted with the help of the DLTS measurements and $I-V$ characteristics at different temperatures, which will be discussed in Sections III-C and III-D.

Fig. 3(b) shows that all four samples exhibit a low leakage current under reverse bias. The reverse $I-V$ curves show a counterclockwise hysteresis, suggesting that the devices trap electrons under high reverse bias. It was within expectations because electrons can be injected from the metal into the $\mathrm{GaN}$ :C layer under the reverse bias that will be captured by traps, as shown in the inset of Fig. 3(b). The electron capture process has been discussed in detail in [22].

\section{DLTS Characteristics}

In Sections III-A and III-B, Run1 and Run2 show very similar $I-V$ and $C-V$ characteristics; therefore, here, we only discuss the DLTS results on samples Run2, Run3, and Run4 to investigate the carrier trapping behavior and the defects existing in the GaN:C layer with different carbon doping concentration. Fig. 4 shows the DLTS spectra for these three samples. Obviously, different DLTS signal shape and polarity are observed for a filling pulse voltage $V_{P}$ at low and high forward bias, respectively. The applied reverse voltage $V_{R}$, the duration of the pulse voltage $T_{P}$, and the rate window $T_{W}$ of the curves are shown in Fig. 4.

Fig. 4(a) reveals that only negative peaks related to electron traps are detected in all three samples when the $V_{P}$ is biased at a low forward voltage $\left(V_{P}=5,5\right.$, and $2 \mathrm{~V}$ for Run2, Run3, and Run4, respectively). It suggests that electron capture is the dominant process at low forward voltage bias. As clearly seen in Fig. 4(a), the electron traps, labeled E1 and E2, are observed in all the three samples. We noticed that the peak height, proportional to the trap concentration, does not scale with the carbon doping concentration, which means that the electron traps E1 and E2 are not carbon-related defects. They most likely correspond with intrinsic defects. The Arrhenius data are obtained by changing the rate of window $T_{W}$. The extracted energy level corresponding to the conduction band edge $\left(E_{C}-E_{T}\right)$ and electron capture cross section $\left(\sigma_{n}\right)$ of E1 are $0.22-0.31 \mathrm{eV}$ and $3 \times 10^{-17}-9 \times 10^{-19} \mathrm{~cm}^{2}$, respectively. A similar energy level and capture cross section for E1 are commonly observed in $\mathrm{GaN}$ grown using different techniques, such as MOCVD [23], HVPE [24], MOVPE [25], and MBE [26]. Alfieri and Sundaramoorthy [27] suggest that this defect is associated with a divacancy $\left(\mathrm{V}_{\mathrm{Ga}}-\mathrm{V}_{\mathrm{N}}\right)$, while Honda et al.'s [28] results have clearly shown that the concentration of this defect is not affected by the presence of impurities, such as 
(a)

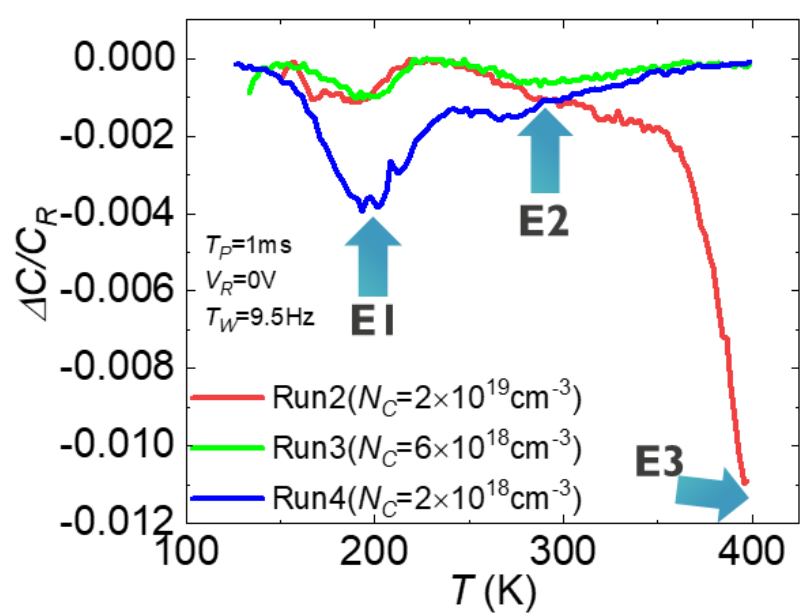

(b)

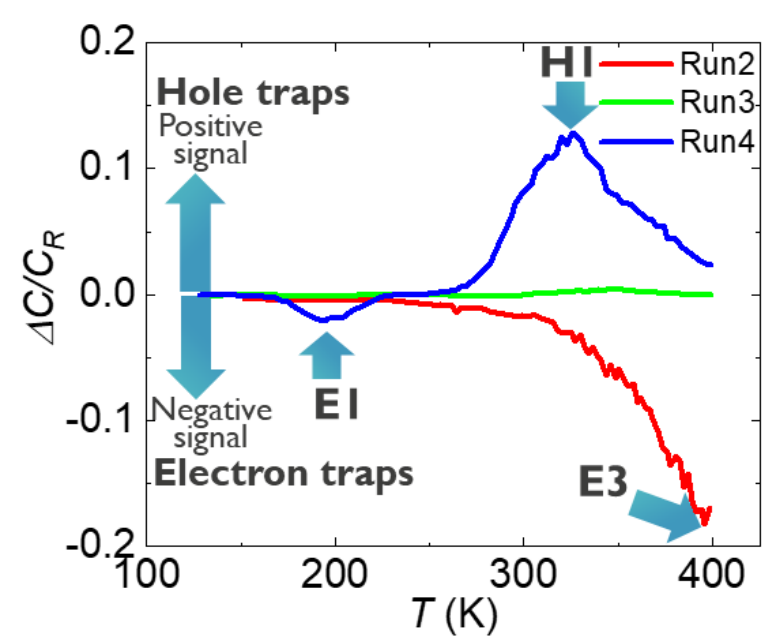

Fig. 4. Temperature-scan DLTS for samples Run2, Run3, and Run4 with (a) low forward pulse voltage and (b) high forward pulse voltage. A $T_{P}$ of $1 \mathrm{~ms}$, a $T_{W}$ of $9.5 \mathrm{~Hz}$, and a $V_{R}$ of $0 \mathrm{~V}$ were used.

carbon. In addition, correlation to a pure edge dislocation [29] and a line defect related to native defects, such as $\mathrm{O}_{\mathrm{N}}$, which are strongly bonded along threading dislocation core sites [30], are also suggested. In our case, by changing the pulse time $T_{P}$ (not shown in here), the trap E1 shows a logarithmic filling behavior and a fixed peak position, suggesting it is an extended defect with localized states [31], [32]. The parameters of the trap E2 cannot be precisely defined due to overlap with the other peaks. Nevertheless, from the literature, we found that a peak similar to E2 located around $300 \mathrm{~K}$ is commonly observed, accompanying E1. For example, both were observed in undoped [33], n-type [29], and carbon-doped GaN layers [34], [35]. The trap E2 is thought to be associated with the $\mathrm{Ga}$ antisite defect $\left(\mathrm{N}_{\mathrm{Ga}}\right)$.

For Run2, another negative peak labeled E3 and located at temperatures over $400 \mathrm{~K}$ can be observed in the DLTS spectrum. We can see that the peak height of E3 should be much higher than for E1 and E2, indicating the trap concentration of E3 is very high, and it should have a significant impact on the device's electrical behavior. E3 is thought to correspond with a C-related defect because the only difference between Run3,

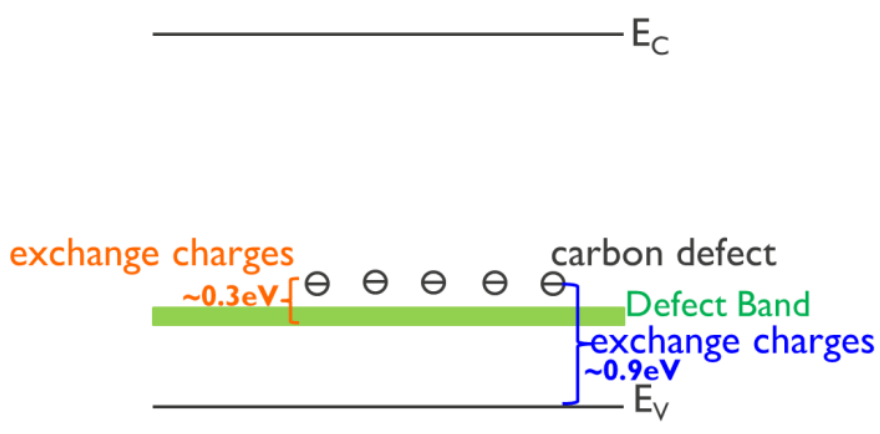

Fig. 5. Schematic of the charge exchange between two defects (orange) instead of between defects and $E_{V}$ (blue).

Run4, and Run2 is the carbon concentration. Note that E3 is only observed in the sample Run2 with a carbon concentration over $2 \times 10^{19} \mathrm{~cm}^{-3}$ and whose $I-V$ curves also show totally different behavior compared with the samples with $N_{C}<$ $2 \times 10^{19} \mathrm{~cm}^{-3}$, implying that the E3 defect begins to dominate the $I-V$ and $C-V$ characteristics. Limited by the measurement equipment, the maximum temperature of the DLTS system is $400 \mathrm{~K}$; therefore, the energy level and capture cross section of E3 cannot be derived yet. However, by monitoring the admittance transient at $1 \mathrm{MHz}$ and at different temperatures, an electron trap with activation energy $\Delta E_{A}=0.29 \mathrm{eV}$ and $\sigma=3 \times 10^{-24} \mathrm{~cm}^{2}$ is extracted (not shown here). The unusual small electron capture cross section indicates that the carrier capture and emission processes may happen between two different defects, instead of between a defect and the valence band edge $\left(E_{V}\right)$, as shown in Fig. 5. In the defect band model proposed by Koller et al. [22], the $\mathrm{C}_{\mathrm{N}}$ deep acceptors exchange charges with a defect band, and carriers can transport along with the defect band. This model fits well with our experimental results.

Fig. 4(b) shows the DLTS spectra of Run2, Run3, and Run4 for a $V_{P}$ bias at a high forward voltage $\left(V_{P}=12\right.$, 10 , and $6 \mathrm{~V}$ for Run2, Run3, and Run4, respectively). For Run3 and Run4 $\left(N_{C}<1 \times 10^{19} \mathrm{~cm}^{-3}\right)$, the devices turn on at the corresponding $V_{P}$, as shown by the $I-V$ curves in Fig. 3, and a positive signal peak, labeled H1, is shown in the DLTS spectrum. This suggests holes exist in the devices and are captured when the device is biased at high $V_{P}$. For the structure in Fig. 1, this hole injection occurs from the metal into the $\mathrm{GaN}$ :C layer under high positive $V_{P}$ conditions. Extracted from the Arrhenius plot, the energy level of $\mathrm{H} 1$ is $0.47 \mathrm{eV}$, with $\sigma=3 \times 10^{-17} \mathrm{~cm}^{2}$, which is associated with the $C_{N}$ induced donor-like traps with $(0 /+)$ state [36]-[39].

For Run2, considering the device reliability, the maximum $V_{P}$ applied is $12 \mathrm{~V}$. The device is under OFF-state at this voltage; therefore, there are a few holes injected into the GaN:C layer, which can be neglected when compared with the electron capture process, resulting in the absence of the positive hole peak in the DLTS spectrum of Run2. In contrast to Run3 and Run4, the negative peak of the E3 defect is observed in the DLTS spectrum of Run2 with $V_{P}=12 \mathrm{~V}$ and the amplitude can be expected higher than the amplitude of H1, indicating there is a large amount of electron capture. 

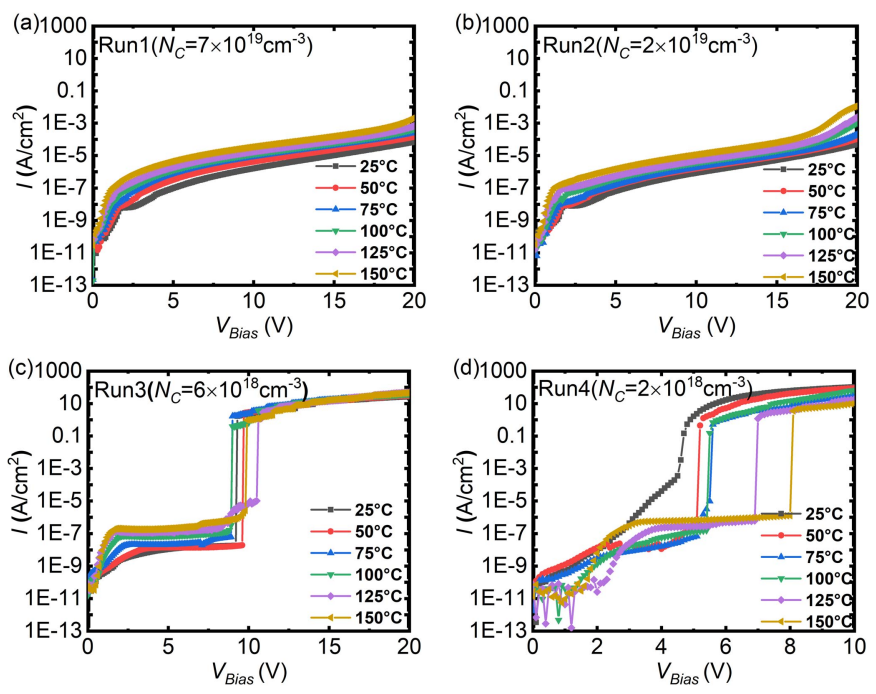

Fig. 6. Forward $I-V$ curves of (a) Run1, (b) Run2, (c) Run3, and (d) Run4.

\section{Carrier Transport Mechanism}

To investigate the carrier transport mechanism in the GaN:C layer with different carbon concentrations, $I-V$ measurements were performed at different temperatures. Fig. 6 shows the forward $I-V$ curves at different temperatures, from $25{ }^{\circ} \mathrm{C}$ to $150{ }^{\circ} \mathrm{C}$ with a temperature step of $25^{\circ} \mathrm{C}$. It is observed that the samples with high (Run1 and Run2) and low (Run3 and Run4) carbon doping concentration show obviously different $I-V$ behavior with temperature changing. Therefore, we will discuss them separately.

1) Samples Run1 and Run $2\left(N_{C}>2 \times 10^{19} \mathrm{~cm}^{-3}\right)$ : The samples Run1 and Run2 show similar $I-V$ characteristics with increasing temperature; here, we will only discuss the carrier transport mechanism for the Run 1 case. The leakage currents of Run1 show a rapid increase from $0.5 \mathrm{~V}$ to around $1.5 \mathrm{~V}$ (depending on the temperature), and then transform to a gradual increase, and therefore, the leakage mechanisms are different in these two regions. Fig. 7(a) shows the log-log plot of the forward $I-V$ curves of Run1. The suddenly increasing currents follow a power-law relation with bias voltage very well:

$$
J \propto V^{m}
$$

which can be modeled by an SCLC mechanism with traps [40]. Before the SCLC current, the transport mechanism is dominated by ohm's law, where the injected carrier density $\left(n_{\text {inj }}\right)$ is lower than the free carrier density $\left(n_{0}\right)$. With bias voltage increasing, $n_{\mathrm{inj}}>n_{0}$, the injected carriers are captured by traps, where the SCLC mechanism may dominate [41].

In the $2-20-\mathrm{V}$ voltage range, the currents gradually increase with increasing bias voltage and are highly dependent on temperature. In this region, P-F emission [42], [43] and variable-range hopping (VRH) [44] may both dominate. The relation between the current density $J$ and the electrical field strength $E$ of the P-F emission mechanism can be
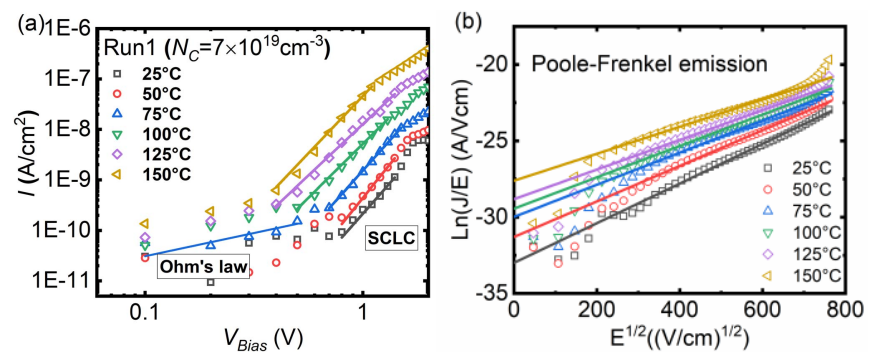

Fig. 7. (a) Log-log $I-V$ curves of Run1. The $m$ used in the fitting is 4-5 for different temperatures. (b) $\ln (J / E)$ versus $\sqrt{E}$ curves of Run1.
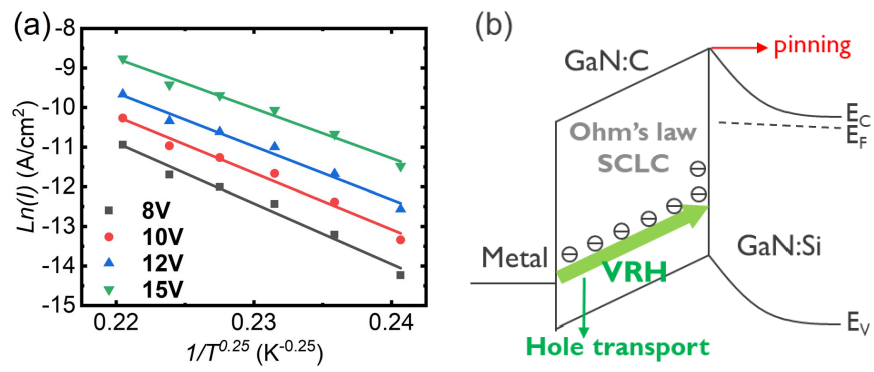

Fig. 8. (a) $\ln (\mathrm{J})$ versus $1 / \mathrm{T}^{0.25}$ curves of Run1 at fixed bias voltage of $8,10,12,15 \mathrm{~V}$. (b) Band diagram and carrier transport mechanism of Run1 and Run2 under forward bias.

expressed as

$$
J \propto \operatorname{Eexp}\left(-\frac{q\left(\varphi_{B}-\sqrt{\frac{q E}{\pi \varepsilon_{r} \varepsilon_{0}}}\right)}{k_{B} T}\right)
$$

where $q \varphi_{B}$ is the trap energy level, $k_{B}$ is Boltzmann's constant, and $T$ is the temperature. Fig. 7(b) shows that the forward current behavior of Run1 follows a good linear relation between $\ln (J / E)$ and $\sqrt{E}$ at all temperatures. The extracted barrier heights by fitting the curves with the P-F emission mechanism using (3) vary from 0.9 to $1.1 \mathrm{eV}$ from $25{ }^{\circ} \mathrm{C}$ to $150{ }^{\circ} \mathrm{C}$. However, the electrical field strength at the bias voltage is low, i.e., $<1 \mathrm{MV} / \mathrm{cm}$, which makes it difficult for carriers to get enough energy to jump over the high barrier. Therefore, VRH could be the dominant mechanism in this region [44], where the conduction follows:

$$
J \propto \exp \left(-\left(\frac{T_{0}}{T}\right)^{\frac{1}{4}}\right) .
$$

Fig. 8(a) shows the forward current at different bias voltage as a function of $1 / T^{0.25}$. The curves are fitted linearly well. The extracted $T_{0}$ is around $4 \times 10^{8} \mathrm{~K}$, which is in good agreement with Koller et al.'s [22] value of $10^{8} \mathrm{~K}$. Besides, the VRH transport mechanism in the GaN buffer layer was commonly found in other articles with carbon doping concentration over $1 \times 10^{19} \mathrm{~cm}^{-3}$ [16], [22]. Fig. 8(b) shows the band diagram and summarizes the carrier transport mechanisms of Run1 and Run2 under forward bias.

2) Samples Run3 and Run4 $\left(N_{C}<1 \times 10^{19} \mathrm{~cm}^{-3}\right)$ : Compared with Run1 and Run2, a turn-on behavior was observed both for Run3 and Run4. Fig. 9 shows the $\log -\log$ scale $I-V$ curves of Run3 and Run4. Here, we give a detailed discussion for the sample Run3. 

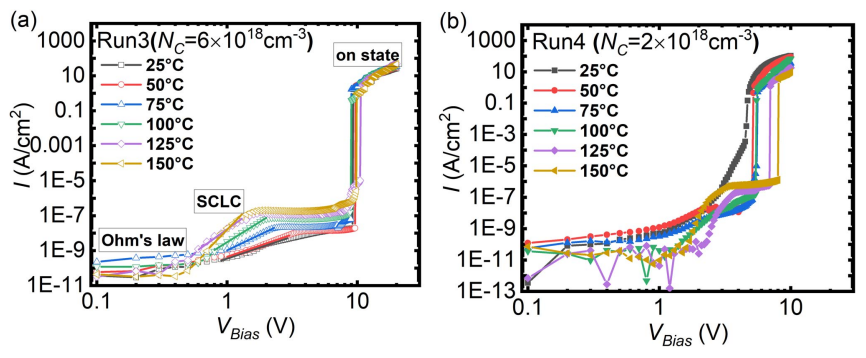

Fig. 9. Forward $I-V$ curves of (a) Run3 and (b) Run4 in log-log scales at different temperature. The carrier transport mechanisms in each region of Run3 are labeled. (a)

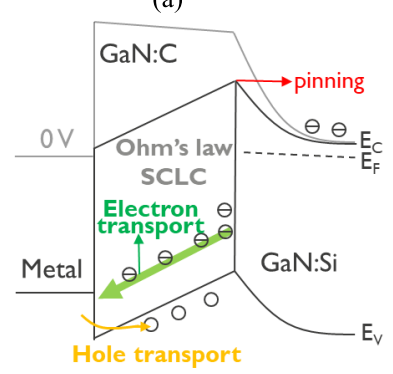

(b)

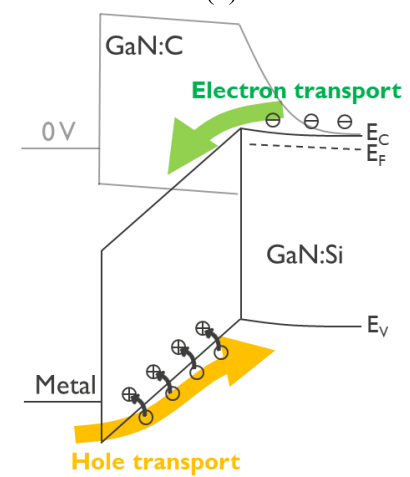

Fig. 10. Band diagram and carrier transport mechanisms (a) before and (b) after the device turning on.

In the $0-0.7-\mathrm{V}$ bias voltage range, the current behavior follows ohm's law, exhibiting a linear relation on a $\log$ $\log$ scale. In the voltage range from 0.7 to $2 \mathrm{~V}$, the current increases quickly and shows a power-law relation with bias voltage, which can also be modeled by an SCLC mechanism with traps. When the bias voltage rises above $2 \mathrm{~V}$, the slope of the current suddenly decreases, which means another transport mechanism comes into play. In the $2-10-\mathrm{V}$ voltage range, the current keeps almost unchanged with increasing bias voltage and just depends on temperature. This can be interpreted by the band diagram in Fig. 10(a). As shown by the $I-V$ hysteresis and DLTS results, electrons are trapped in defects under this forward bias. The current in this voltage range is dominated by the carrier transport between defects. We consider that the defects (located at the interface of $\mathrm{GaN}: \mathrm{C} / \mathrm{GaN}: \mathrm{Si}$ or in the $\mathrm{GaN}: \mathrm{C}$ layer near the interface) are filled by electrons, leading to a potential barrier. This phenomenon is commonly named the pinning effect. Therefore, the electrons in the GaN:Si layer and the holes in the $\mathrm{GaN}: \mathrm{C}$ layer have to overcome the barrier to contribute to the current. With the bias increasing, more holes are injected into GaN:C layer from metal. Some defects begin to capture holes, causing the barrier height to decrease quickly. The electrons in the GaN:Si layer and the holes in the $\mathrm{GaN}: \mathrm{C}$ layer then can easily overcome the barrier, and the device turns on steeply. In addition, despite the holes capture mechanism, we would like to notice that other trap- and fieldrelated mechanisms can be considered leading to the device turning on [21].

When the device turns on, a high density of holes is injected into the $\mathrm{GaN}: \mathrm{C}$ layer and captured by the donor-like traps in the GaN:C bulk. This is supported by the observed high positive peak (hole trap H1) in the DLTS spectra when $V_{P}$ is biased at high forward voltage [Fig. 4(b)] and the big counterclockwise $I-V$ hysteresis in Fig. 3(a). Fig. 10(b) shows the band diagram and carrier transport mechanisms after the device turn-on.

The sample Run4 $\left(N_{C}=2 \times 10^{18} \mathrm{~cm}^{-3}\right)$ shows a weak current blocking capability at forward bias voltage. The leakage current exhibits similar behavior with Run3, despite a short-voltage-independent region and earlier device conduction, which results from a lower defect concentration to pin the Fermi level.

\section{CONCLUSION}

In this work, a Metal/GaN:C/GaN:Si structure is used to investigate the defect charging process and carrier transport mechanisms in the GaN layer with different carbon doping concentration. Obviously, different forward $I-V$ behavior is observed between the samples with high $\left(N_{C}>\right.$ $\left.1 \times 10^{19} \mathrm{~cm}^{-3}\right)$ and low $\left(N_{C}<1 \times 10^{19} \mathrm{~cm}^{-3}\right)$ carbon concentration. For the samples with high carbon concentration, Run1 and Run2, Ohm's law, SCLC, and VRH may dominate the carrier transport mechanism in sequence when increasing the forward bias. For the samples with low carbon concentration, Run3 and Run4, besides the above carrier transport mechanism, a sudden device turning on behavior was observed. In addition, it is found that carbon introduces electron traps at or near the $\mathrm{GaN}: \mathrm{C} / \mathrm{GaN}: \mathrm{Si}$ interface, which results in a strong pinning effect and builds up a barrier impeding carrier injection. Different DLTS spectra are also observed between samples with high and low carbon concentration. The samples with high $N_{C}$ exhibit a high density of electron traps in the whole forward bias range, which is interpreted by defects exchanging charges with a defect band. While the samples with low carbon doping concentration show an electron trap at low bias voltage and a hole trap at high bias voltage, resulting from defects exchanging charges with the valence band.

\section{ACKNOWLEDGMENT}

The authors would like to thank the fruitful discussions with Dr. Christian Koller and Dr. Clemens Ostermaier.

\section{REFERENCES}

[1] K.-T. Lee et al., "GaN devices on a $200 \mathrm{~mm}$ Si platform targeting heterogeneous integration," IEEE Electron Device Lett., vol. 38, no. 8, pp. 1094-1096, Aug. 2017, doi: 10.1109/LED.2017.2720688.

[2] R. A. Khadar, C. Liu, R. Soleimanzadeh, and E. Matioli, "Fully vertical GaN-on-Si power MOSFETs," IEEE Electron Device Lett., vol. 40, no. 3, pp. 443-446, Mar. 2019, doi: 10.1109/LED.2019.2894177.

[3] X. Li et al., "Demonstration of $\mathrm{GaN}$ integrated half-bridge with on-chip drivers on 200-mm engineered substrates," IEEE Electron Device Lett., vol. 40, no. 9, pp. 1499-1502, Sep. 2019, doi: 10.1109/LED.2019.2929417.

[4] M. Zhao et al., "Growth and characterization of DH-HEMT structures with various $\mathrm{AlGaN}$ barriers and AlN interlayers on $200 \mathrm{~mm} \mathrm{Si(111)}$ substrates," Phys. Status Solidi (C), vol. 11, nos. 3-4, pp. 446-449, Feb. 2014, doi: 10.1002/pssc.201300478.

[5] N. E. Posthuma et al., "An industry-ready $200 \mathrm{~mm} \mathrm{p-GaN} \mathrm{E-}$ mode GaN-on-Si power technology," in Proc. IEEE 30th Int. Symp. Power Semiconductor Devices ICs (ISPSD), May 2018, pp. 284-287, doi: 10.1109/ISPSD.2018.8393658.

[6] A. Fariza et al., "Leakage currents and Fermi-level shifts in GaN layers upon iron and carbon-doping," J. Appl. Phys., vol. 122, no. 2, Jul. 2017, Art. no. 025704, doi: 10.1063/1.4993180. 
[7] M. Singh et al., "Lateral charge spreading and device-to-device coupling in C-doped AlGaN/GaN-on-Si wafers," Microelectron. Rel., vol. 95, pp. 81-86, Apr. 2019, doi: 10.1016/j.microrel.2019.02.012.

[8] C. H. Seager, D. R. Tallant, J. Yu, and W. Götz, "Luminescence in GaN co-doped with carbon and silicon," J. Lumin., vol. 106, no. 2, pp. 115-124, Mar. 2004, doi: 10.1016/j.jlumin.2003.08.004.

[9] D. S. Green, U. K. Mishra, and J. S. Speck, "Carbon doping of GaN with $\mathrm{CBr}_{4}$ in radio-frequency plasma-assisted molecular beam epitaxy," J. Appl. Phys., vol. 95, no. 12, pp. 8456-8462, Jun. 2004 doi: 10.1063/1.1755431.

[10] R. Armitage, Q. Yang, and E. R. Weber, "Analysis of the carbon-related 'blue' luminescence in GaN," J. Appl. Phys., vol. 97, no. 7, Mar. 2005, Art. no. 073524, doi: 10.1063/1.1856224.

[11] C. H. Seager, A. F. Wright, J. Yu, and W. Götz, "Role of carbon in GaN," J. Appl. Phys., vol. 92, no. 11, pp. 6553-6560, Dec. 2002, doi: 10.1063/1.1518794.

[12] H. Yacoub et al., "Effect of different carbon doping techniques on the dynamic properties of GaN-on-Si buffers," IEEE Trans. Electron Devices, vol. 64, no. 3, pp. 991-997, Mar. 2017, doi: 10.1109/TED.2017.2647841.

[13] Y. Zhang et al., "GaN-on-Si vertical Schottky and p-n diodes," IEEE Electron Device Lett., vol. 35, no. 6, pp. 618-620, Jun. 2014, doi: 10.1109/LED.2014.2314637.

[14] P. Moens et al., "Impact of buffer leakage on intrinsic reliability of 650 V AlGaN/GaN HEMTs," in IEDM Tech. Dig., Dec. 2015, p. 35 , doi: 10.1109/IEDM.2015.7409831.

[15] C. Zhou, Q. Jiang, S. Huang, and K. J. Chen, "Vertical leakage/breakdown mechanisms in AlGaN/GaN-on-Si devices," IEEE Electron Device Lett., vol. 33, no. 8, pp. 1132-1134, Aug. 2012, doi: 10.1109/LED.2012.2200874.

[16] D. C. Look et al., "Deep-center hopping conduction in GaN," J. Appl. Phys., vol. 80, no. 5, pp. 2960-2963, Sep. 1996, doi: 10.1063/1.363128.

[17] S. Dalcanale, M. J. Uren, and M. Kuball, "TCAD simulation of leakage through threading dislocations in GaN-based pn-diodes," Simul. Standard, vol. 28, no. 3, pp. 7-8, Sep. 2018.

[18] M. J. Uren, M. Cäsar, M. A. Gajda, and M. Kuball, "Buffer transport mechanisms in intentionally carbon doped GaN heterojunction field effect transistors," Appl. Phys. Lett., vol. 104, no. 26, Jun. 2014, Art. no. 263505, doi: 10.1063/1.4885695.

[19] H. Yacoub et al., "The effect of the inversion channel at the AlN/Si interface on the vertical breakdown characteristics of GaN-based devices," Semicond. Sci. Technol., vol. 29, no. 11, Nov. 2014, Art. no. 115012 , doi: 10.1088/0268-1242/29/11/115012.

[20] C. Koller, G. Pobegen, C. Ostermaier, M. Huber, and D. Pogany, "The interplay of blocking properties with charge and potential redistribution in thin carbon-doped GaN on n-doped GaN layers," Appl. Phys. Lett., vol. 111, no. 3, Jul. 2017, Art. no. 032106, doi: 10.1063/1.4993571.

[21] C. Koller et al., "Trap-related breakdown and filamentary conduction in carbon doped GaN," Phys. Status Solidi (B), vol. 256, no. 6, Jun. 2019, Art. no. 1800527, doi: 10.1002/pssb.201800527.

[22] C. Koller, G. Pobegen, C. Ostermaier, and D. Pogany, "Effect of carbon doping on charging/discharging dynamics and leakage behavior of carbon-doped GaN," IEEE Trans. Electron Devices, vol. 65, no. 12, pp. 5314-5321, Dec. 2018, doi: 10.1109/TED.2018.2872552.

[23] Y. Tokuda, Y. Matsuoka, H. Ueda, O. Ishiguro, N. Soejima, and T. Kachi, "DLTS study of n-type GaN grown by MOCVD on GaN substrates," Superlattices Microstruct., vol. 40, nos. 4-6, pp. 268-273, Oct. 2006, doi: 10.1016/j.spmi.2006.07.025.

[24] P. Hacke, T. Detchprohm, K. Hiramatsu, N. Sawaki, K. Tadatomo, and K. Miyake, "Analysis of deep levels in $n$-type GaN by transient capacitance methods," J. Appl. Phys., vol. 76, no. 1, pp. 304-309, Jul. 1994, doi: $10.1063 / 1.357144$.

[25] E. Płaczek-Popko, J. Trzmiel, E. Zielony, S. Grzanka, R. Czernecki, and T. Suski, "Deep level transient spectroscopy signatures of majority traps in $\mathrm{GaN}$ p-n diodes grown by metal-organic vapor-phase epitaxy technique on GaN substrates," Phys. B, Condens. Matter, vol. 404, nos. 23-24, pp. 4889-4891, 2009, doi: 10.1016/j.physb.2009.08.237.

[26] C. D. Wang et al., "Deep level defects in n-type GaN grown by molecular beam epitaxy," Appl. Phys. Lett., vol. 72, no. 10, pp. 1211-1213, Mar. 1998, doi: 10.1063/1.121016.
[27] G. Alfieri and V. K. Sundaramoorthy, "Defect energy levels in carbon implanted n-type homoepitaxial GaN," J. Appl. Phys., vol. 126, no. 12 Sep. 2019, Art. no. 125301, doi: 10.1063/1.5109237.

[28] U. Honda, Y. Yamada, Y. Tokuda, and K. Shiojima, "Deep levels in n-GaN doped with carbon studied by deep level and minority carrier transient spectroscopies," Jpn. J. Appl. Phys., vol. 51, no. 4S, Apr. 2012, Art. no. 04DF04, doi: 10.1143/JJAP.51.04DF04.

[29] H. K. Cho, C. S. Kim, and C.-H. Hong, "Electron capture behaviors of deep level traps in unintentionally doped and intentionally doped ntype GaN," J. Appl. Phys., vol. 94, no. 3, pp. 1485-1489, Jul. 2003, doi: $10.1063 / 1.1586981$

[30] C. B. Soh, S. J. Chua, H. F. Lim, D. Z. Chi, S. Tripathy, and W. Liu, "Assignment of deep levels causing yellow luminescence in GaN," J. Appl. Phys., vol. 96, no. 3, pp. 1341-1347, Aug. 2004, doi: $10.1063 / 1.1757654$

[31] W. Schröter and H. Cerva, "Interaction of point defects with dislocations in silicon and germanium: Electrical and optical effects," Solid State Phenomena, vols. 85-86, pp. 67-144, Dec. 2001, doi: 10.4028/www.scientific.net/ssp.85-86.67.

[32] W. Schröter, J. Kronewitz, U. Gnauert, F. Riedel, and M. Seibt, "Bandlike and localized states at extended defects in silicon," Phys. Rev. B, Condens. Matter, vol. 52, no. 19, pp. 13726-13729, Nov. 1995, doi: 10.1103/PhysRevB.52.13726.

[33] T. T. Duc, G. Pozina, E. Janzén, and C. Hemmingsson, "Investigation of deep levels in bulk GaN material grown by halide vapor phase epitaxy," J. Appl. Phys., vol. 114, no. 15, Oct. 2013, Art. no. 153702, doi: $10.1063 / 1.4825052$.

[34] T. Tanaka, K. Shiojima, Y. Otoki, and Y. Tokuda, "A study on multiple defect states in low-carbon doped GaN layers and its correlation with AlGaN/GaN high electron mobility transistor operation," Thin Solid Films, vol. 557, pp. 207-211, Apr. 2014, doi: 10.1016/j.tsf.2013. 10.077 .

[35] A. Armstrong et al., "Impact of carbon on trap states in n-type GaN grown by metalorganic chemical vapor deposition," Appl. Phys. Lett., vol. 84, no. 3, pp. 374-376, Jan. 2004, doi: 10.1063/1.1643540.

[36] J. L. Lyons, A. Janotti, and C. G. Van de Walle, "Effects of carbon on the electrical and optical properties of InN, GaN, and AlN," Phys. Rev. B, Condens. Matter, vol. 89, no. 3, p. 35204, Jan. 2014, doi: 10.1103/PhysRevB.89.035204.

[37] M. A. Reshchikov, D. O. Demchenko, A. Usikov, H. Helava, and Y. Makarov, "Carbon defects as sources of the green and yellow luminescence bands in undoped GaN," Phys. Rev. B, Condens. Matter, vol. 90 , no. 23, Dec. 2014, Art. no. 235203, doi: 10.1103/PhysRevB.90. 235203.

[38] D. O. Demchenko, I. C. Diallo, and M. A. Reshchikov, "Yellow luminescence of gallium nitride generated by carbon defect complexes," Phys. Rev. Lett., vol. 110, no. 8, Feb. 2013, Art. no. 087404, doi: 10.1103/PhysRevLett.110.087404

[39] T. Narita, K. Tomita, Y. Tokuda, T. Kogiso, M. Horita, and T. Kachi, "The origin of carbon-related carrier compensation in p-type GaN layers grown by MOVPE," J. Appl. Phys., vol. 124, no. 21, Dec. 2018, Art. no. 215701, doi: 10.1063/1.5057373.

[40] M. A. Lampert, "Simplified theory of space-charge-limited currents in an insulator with traps," Phys. Rev., vol. 103, no. 6, pp. 1648-1656, Sep. 1956, doi: 10.1103/PhysRev.103.1648.

[41] A. Hinoki et al., "Effects of traps formed by threading dislocations on off-state breakdown characteristics in $\mathrm{GaN}$ buffer layer in $\mathrm{AlGaN} / \mathrm{GaN}$ heterostructure field-effect transistors," Appl. Phys. Express, vol. 1, no. 1, Dec. 2007, Art. no. 011103, doi: 10.1143/APEX.1.011103.

[42] A. Uedono, M. Zhao, and E. Simoen, "Probing the effect of point defects on the leakage blocking capability of $\mathrm{Al}_{0.1} \mathrm{Ga}_{0.9} \mathrm{~N} / \mathrm{Si}$ structures using a monoenergetic positron beam," J. Appl. Phys., vol. 120, no. 21, Dec. 2016, Art. no. 215702, doi: 10.1063/1.4970984.

[43] W. Zhang, E. Simoen, M. Zhao, and J. Zhang, "Analysis of leakage mechanisms in AlN nucleation layers on p-Si and p-SOI substrates," IEEE Trans. Electron Devices, vol. 66, no. 4, pp. 1849-1855, Apr. 2019, doi: 10.1109/TED.2019.2899964.

[44] X. Li et al., "Buffer vertical leakage mechanism and reliability of 200-mm GaN-on-SOI," IEEE Trans. Electron Devices, vol. 66, no. 1, pp. 553-560, Jan. 2019, doi: 10.1109/TED.2018.2878457. 